\title{
The Use of Body Surface Potential Map for Identifying Sites of Accessory Pathway in Patients with Wolff-Parkinson-White Syndrome
}

\author{
Yung-Zu Tseng, MD, FCCP, Kwan-Lih Hsu, MD, PhD, \\ Fu-Tien Chiang, MD, PhD, Huey-Ming Lo, MD, \\ Chuen-Den Tseng, MD, PhD, \\ and Jiunn-Lee LiN, MD, PhD
}

\section{SUMMARY}

The body surface potential map (BSPM) may reflect regional myocardial electrical activity. This technique can thus provide information regarding the excitation of ventricles. This study is an attempt to evaluate the usefulness of BSPM in determining the sites of the atrioventricular (AV) accessory pathway (AP) in patients with Wolff-Parkinson-White (W-P-W) syndrome.

The BSPMs were obtained from 40 consecutive patients with W-P-W syndrome in a fasting state, using the heart potential map system designed by Toyama et al. Unipolar electrocardiograms were recorded simultaneously from 87 lead points on the chest surface, including 59 lead points on the anterior chest and 28 on the back. Wilson's central terminal was used as a voltage reference and BSPMs in an isopotential distribution pattern were made every millisecond throughout ventricular activation from these unipolar ECGs with the use of a microcomputer system.

All patients underwent an electrophysiologic study (EPS) at cardiac catheterization. We analyzed the potential distribution during ventricular depolarization and compared the results between EPS and BSPM findings.

The following results were obtained: (1) seven types of BSPM pattern were identified in accordance with the sites of the AV AP confirmed by EPS; (2) the location of the potential minimum of ventricular depolarization and the direction of the excitation wavefront during early ventricular depolarization, the reversal pattern of ventricular potential distribution, the epicardial right ventricular breakthrough and the dynamic change of ventricular potential distribution were useful for the detection of the ventricular pre-excitation site; (3) epicardial right ventricular breakthrough occurred in nearly all patients with left ventricular free wall accessory AV connections; (4) the abnormal early reversal pattern of ventricular potential distribution did not occur in patients with left ventricular AV connections but did appear in most patients with right

From the Department of Internal Medicine, College of Medicine, National Taiwan University, Taipei, Taiwan.

Address for correspondence: Yung-Zu Tseng, MD, FCCP, Department of Internal Medicine, National

Taiwan University Hospital, 7, Chung-Shan South Road, Taipei 100, Taiwan.

Received for publication November 26, 1997.

Revised and accepted May 8, 1998. 
ventricular free wall AV connections. Accordingly, BSPM is a reliable noninvasive procedure to determine the ventricular pre-excitation sites of patients with W-P-W syndrome. (Jpn Heart J 1998; 39: 445-455)

Key words: Potential minimum, Potential maximum, Right ventricular breakthrough, W-P-W syndrome

$\mathrm{W}$

OLFF-PARKINSON-WHITE (W-P-W) syndrome is considered to be caused by the early excitation of some ventricular sites through accessory atrio-ventricular (AV) pathways. ${ }^{1)}$ The accessory pathway (AP) can exist anywhere along the AV ring except where the left atrium is attached to the aortic ring. ${ }^{2)}$ Identification and localization of the AP in patients with this syndrome may be imperative for management. This is particularly true for patients with significant tachyarrhythmia requiring interruption of the APs. A reliable noninvasive procedure to determine the location of APs in patients with this syndrome would be clinically useful. The body surface potential map (BSPM) may reflect regional myocardial electrical activity and can provide information regarding the ventricular excitation which can not be derived from conventional electrocardiography. ${ }^{\left.3{ }^{34}\right)}$ This technique has been reported to be potentially applicable in identifying the location of APs in patients with W-P-W syndrome. ${ }^{5-17)}$ However, the identifying parameter remains to be established.

In this report, we will present the BSPM data which are useful in identifying the site of the AP in patients with W-P-W syndrome.

\section{Materials and Methods}

Forty consecutive patients with W-P-W syndrome were studied. Their ages ranged from 16 years to 70 years. There were 28 males and 12 females. Electrophysiological (EPS) studies at cardiac catheterization were performed after BSPM recording to identify the earliest ventricular activation. The BSPM was obtained in a fasting state using the heart potential map system designed by Toyama et al. ${ }^{\text {18) }}$ (HPM-6500 Fukuda Denshi Co., Ltd., Nagoya, Japan). Unipolar electrocardiograms were recorded simultaneously from 87 leads points on the chest surface. There were 59 lead points on the anterior chest wall and 28 on the back. The positions of electrodes in this system are shown in Figure 1. The points A, I and $\mathrm{E}$ were at the right mid-axillary line, left mid-axillary line and mid-sternal line, respectively. The distance from $A$ to $E$ was divided into 4 equal parts and the positions of lead points $\mathrm{B}, \mathrm{C}$ and $\mathrm{D}$ were thus ascertained. Similarly, the distance from $\mathrm{E}$ to $\mathrm{I}$ was also divided into 4 equal parts and the points $\mathrm{F}, \mathrm{G}$ and $\mathrm{H}$ were positioned. Points $\mathrm{J}$ and $\mathrm{M}$ were determined at the same interval using the method for determining points described above. The distance from $\mathrm{J}$ to $\mathbf{M}$ was 


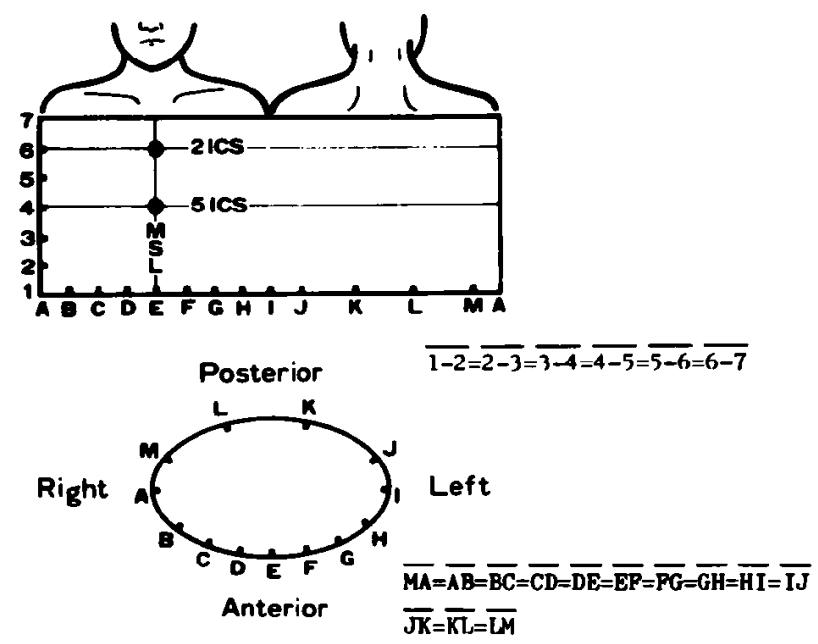

Figure 1. Position of electrodes in the recording system of the body surface potential map.

divided into 3 equal parts and the positions of lead points $\mathrm{K}$ and $\mathrm{L}$ were ascertained. Accordingly, the anterior chest wall was divided into 8 columns (from $\mathrm{A}$ to $I$ ) and the back into 5 columns (from I to $A$ ). In addition, the thorax was divided horizontally into 7 rows. The point E6 was set at the level of the 2nd intercostal space, and E4 at the 5th intercostal space. The position of E5 was centered between E4 and E6. The points E1, E2, E3 and E7 were set at the same intervals as those between $\mathrm{E} 6$ and $\mathrm{E} 5$ or $\mathrm{E} 4$ and $\mathrm{E} 5$.

Wilson's central terminal was used as a voltage reference and BSPMs were made in an isopotential distribution pattern every millisecond throughout ventricular activation from these unipolar ECGs using a microcomputer system. To illustrate the BSPMs, a planar map was drawn with a cut along the right midaxillary line. The solid line illustrated an equipotential line at an interval of 0.4 $\mathrm{mV}$. The line with zero potential, representing the potential of Wilson's central terminal, was called the zero line. The symbol " + " indicates a potential maximum and the symbol "-_" indicates a potential minimum. We analyzed the potential distribution during ventricular depolarization. The parameters for analysis included potential distribution in the early period of ventricular depolarization, the epicardial right ventricular breakthrough (RVBT) and dynamic change of potential distribution.

We studied the relationship between BSPM findings and sites of AP as confirmed by EPS at catheterization. 


\section{Results}

Location of accessory pathway: A single AP was present in all 40 patients. These 40 patients were classified into 7 groups according to the pre-excited sites as determined by EPS at cardiac catheterization (Figure 2). There were 9 patients with anterior right ventricular (RV) AP in group I; 12 with lateral RV AP in group II; 2 with posterior RV AP in group III; 4 with posteroseptal AP in group IV; 5 with posterior left ventricular (LV) AP in group V; 5 with lateral LV AP in group VI; and 3 with anterior LV AP in group VII.

The potential distribution on the thorax of patients with Wolff-ParkinsonWhite syndrome during the early period of ventricular depolarization: There were 7 types of potential distribution patterns during the early period of ventricular depolarization (the initial $40 \mathrm{msec}$ period with constant potential distribution). This classification was made in accordance with the anatomic site of $\mathrm{APs}$ along the A-V ring as shown in Figure 2.

In patients with the AP in the anterior $R V$, the potential minimum was located higher than the potential maximum during the initial period of the QRS complex. The potential minimum appeared in the middle or right superior portion of the forechest and the potential maximum was in the left-inferior portion of the thorax, either anterior or posterior (type I BSPM pattern, Figure 3A). An example is illustrated in Figure 4A.

In patients with the AP in the lateral $R V$, the potential minimum was

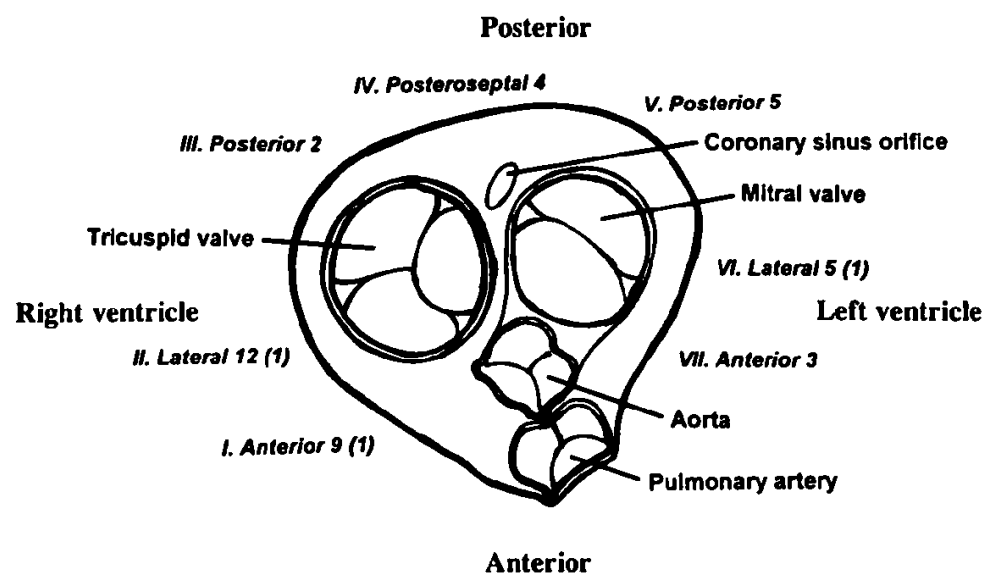

Figure 2. Classification of accessory pathway based on anatornic subdivisions along the atrioventricular ring. Figures at anatomic sites indicate the number of patients. Figures in parentheses indicate the number of patients who underwent surgery for accessory pathways. 
A

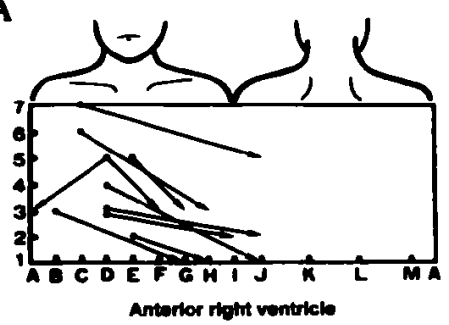

B

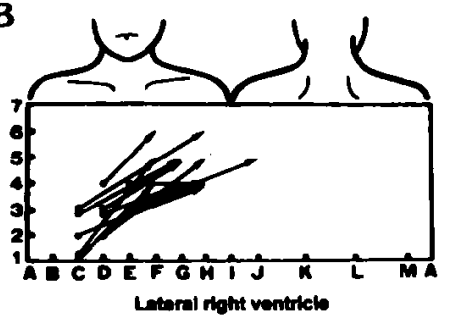

C

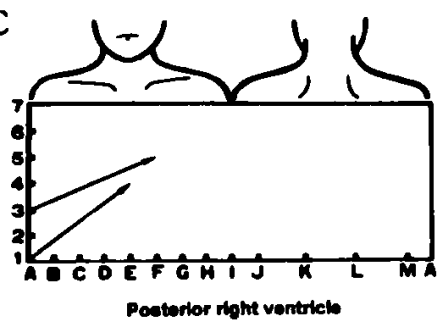

D

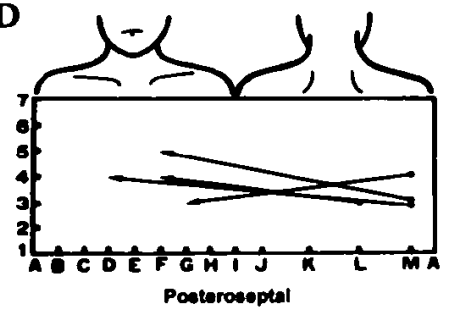

$\mathbf{E}$

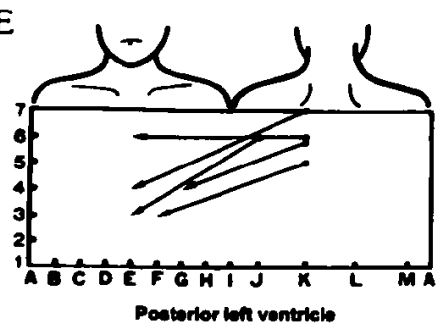

$\mathbf{F}$

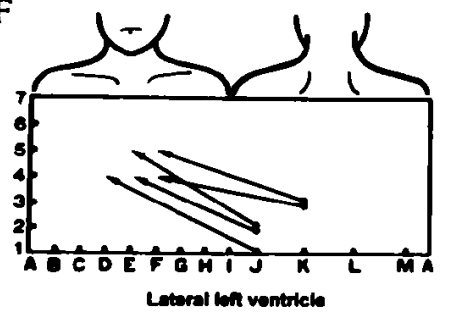

$\mathbf{G}$

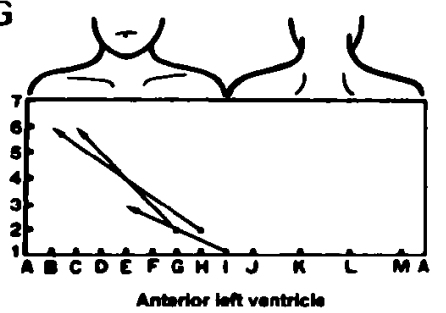

Figure 3. The potential distribution of Wolff-Parkinson-White syndrome during the initial period of ventricular depolarization.

located lower than the potential maximum during the initial period of the QRS complex. The potential minimum was found in the middle or right-inferior portion of the forechest, while the potential maximum was located in the left-superior portion of the forechest (type II BSPM pattern, Figure 3B). An example is shown in Figure 4B.

In patients with the $\mathrm{AP}$ in the posterior $\mathrm{RV}$, the potential minimum was located lower than the potential maximum during the initial period of the QRS complex. The potential minimum appeared in the inferior portion of the right 
A

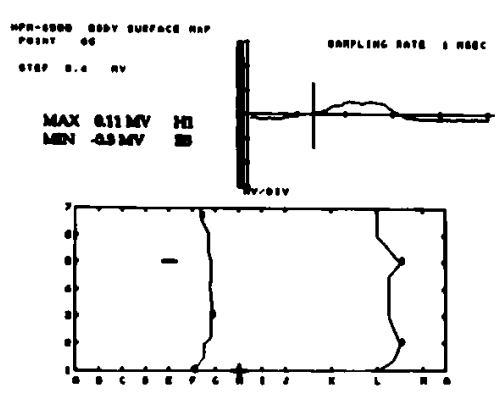

B

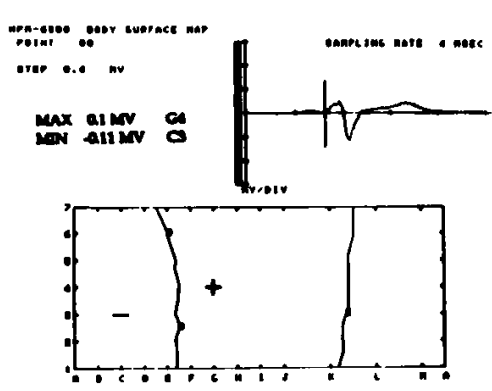

C

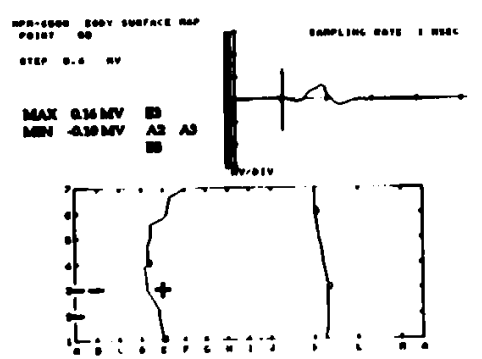

D

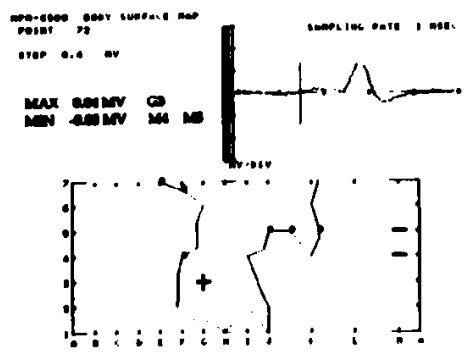

E

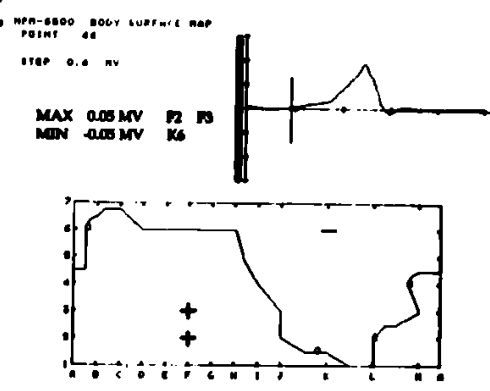

F

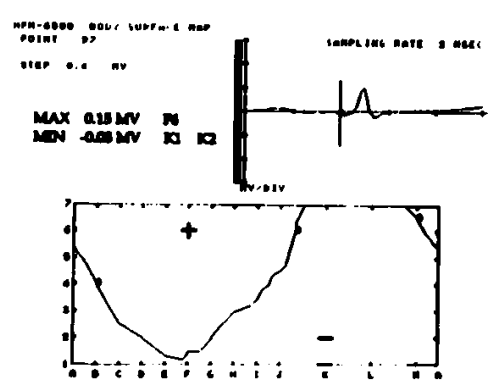

G

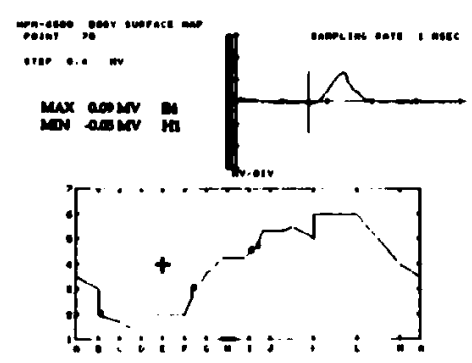

Figure 4. Examples of the potential distribution of Wolff-Parkinson-White syndrome during the initial period of ventricular depolarization. 
Table. Various Parameters of Body Surface Potential Map in Patients with Wolff-Parkinson-White Syndrome

\begin{tabular}{|c|c|c|c|c|c|}
\hline \multirow{2}{*}{\multicolumn{2}{|c|}{$\begin{array}{c}\text { BSPM } \\
\text { classification }\end{array}$}} & \multicolumn{2}{|c|}{ Right ventricular breakthrough } & \multicolumn{2}{|c|}{$\begin{array}{c}\text { Abnormal early reversal pattern of } \\
\text { ventricular depolarization }\end{array}$} \\
\hline & & \multirow{2}{*}{$\begin{array}{c}\text { No. } \\
0\end{array}$} & \multirow{2}{*}{$\begin{array}{c}\% \\
0\end{array}$} & \multirow{2}{*}{$\frac{\text { No. }}{8}$} & \multirow{2}{*}{$\frac{\%}{88.9}$} \\
\hline I & (9) & & & & \\
\hline & (12) & 0 & 0 & 10 & 83.3 \\
\hline III & (2) & 0 & 0 & 2 & 100.0 \\
\hline IV & (4) & 4 & 100.0 & 0 & 0 \\
\hline V & (5) & 4 & 80.0 & 0 & 0 \\
\hline $\mathrm{VI}$ & (5) & 5 & 100.0 & 0 & 0 \\
\hline VII & (3) & 3 & 100.0 & 0 & 0 \\
\hline
\end{tabular}

BSPM = body surface potential map. Figures in parentheses indicate the numbers of patients per group

axilla and did the potential maximum in the middle portion of the forechest (type III BSPM pattern, Figure 3C). An illustration is shown in Figure 4C.

In patients with the AP in the posteroseptum, the potential minimum was slightly lower than the potential maximum in all cases except one, during the initial period of the QRS complex. The potential minimum was found in the right-inferior portion of the back and the potential maximum was in the middle portion of the forechest in all cases except one (type IV BSPM pattern, Figure 3D). An illustration is given in Figure 4D.

In the group with the AP in the posterior $L V$, the potential minimum was located higher than the potential maximum during the initial period of the QRS complex. The potential minimum appeared in the middle-superior portion or left-superior portion of the back while the potential maximum was in the middle portion of the forechest (type V BSPM pattern, Figure 3E). An example is shown in Figure 4E.

In the group with the AP in the lateral $L V$, the potential minimum was located lower than the potential maximum during the initial period of the QRS complex. The potential minimum appeared in the left-inferior portion of the back while the potential maximum was found in the middle portion of the forechest (type VI BSPM pattern, Figure 3F). An example is shown in Figure 4F.

In patients with the $A P$ in the anterior $L V$, the potential minimum was located lower than the potential maximum during the initial period of the QRS complex. The potential minimum was found in the left-inferior portion of the forechest and the potential maximum appeared in the right-superior portion of the forechest (type VII BSPM pattern, Figure 3G). An example is shown in Figure 4G.

Various parameters of body surface potential maps (Table): There were differences in various parameters of the absolute value of the BSPMs between 
right-sided and left-sided APs.

None of the patients with RV APs demonstrated RVBT. However, the RVBT was found in 16 of 17 patients with LV APs.

An abnormal early reversal pattern of ventricular depolarization in which the absolute value of the potential minimum was greater than that of the potential maximum during the early phase of ventricular depolarization (the initial 40 msec period with constant potential distribution), ${ }^{4)}$ was observed in 20 of 23 patients with the RV AP, but not in patients with LV AP.

\section{Discussion}

This study identified 7 types of BSPM according to the sites of APs determined by EPS. Yamada et al. ${ }^{5)}$ first identified 3 types of BSPM in patients with W-P-W syndrome, according to the potential distribution during the QRS complex. There then followed several reports addressing the question of the usefulness of BSPM in identifying the insertion site of the accessory A-V pathway. De Ambroggi et al. ${ }^{6}$ classified 6 types of BSPM based on the location of the potential minimum and potential maximum during the delta wave. Iwa et al. ${ }^{8)}$ observed 5 types of BSPM according to the location of the potential minimum at 40 milliseconds of the QRS complex and stressed the value of this criterion in predicting the sites of APs. Benson et al. ${ }^{9)}$ observed 8 types of BSPM according to the different sites of APs while Kamakura et al. ${ }^{11)}$ found 7 types. Liebman et al. ${ }^{12)}$ observed 6 types of BSPM and emphasized the value of the QRS map and RVBT in predicting the location of APs.

Several indices of BSPM were considered as useful criteria in predicting the site of APs. Iwa et al. ${ }^{8)}$ did not accept the analysis of the potential distribution during 40 milliseconds after the onset of the QRS complex as a reliable criterion for determining the location of APs in patients with W-P-W syndrome. Benson $t$ al. ${ }^{9)}$ reported the predictive reliability of potential distribution in the first 40 milliseconds of the QRS complex for the sites of APs. Kamakura et al. ${ }^{11)}$ found that stability of the QRS potential distribution during the delta wave always occurred both in the initial 40 milliseconds and at a peak negative potential of -150 to $-200 \mu \mathrm{V}$. They therefore recommended that an amplitude-based map rather than a time-based map was useful in localization of APs. The predictive ability of their criterion ranged from 30 to 35 of 41 cases. Liebman et al. ${ }^{12]}$ stressed the importance of both negative and positive, not just negative potentials, and disagreed with the recommendation of Kamakura et al. Benson et al. ${ }^{9)}$ reported the superiority of the ST-T map over the QRS map in predicting the location of APs. Some reports ${ }^{15,17)}$ also demonstrated the close relationship between BSPM of repolarization abnormalities and the location of the accessory 
pathway in patients with manifest W-P-W syndrome. However, Liebman et al. ${ }^{12)}$ documented the usefulness of this potential distribution, pointing out that it provided limited accuracy only in some cases with marked ventricular pre-excitation. It is well known that the potential maximum in a given area on the chest surface represents the area that an intracardiac excitation wavefront is pointing toward. The potential minimum indicates the negative aspect of a wavefront where the excitation is initiated. ${ }^{19)}$ The location of the potential minimum during the delta wave is thus an ideal index for predicting the pre-excitation site of APs and has proven to be useful in the previous literature, and in our report. Liebman et al. ${ }^{12}$ even found that the ventricular insertion sites of APs determined by QRS analysis of BSPM and surgical EPS mapping were identical or within $1.5 \mathrm{~cm}$ in all of their cases with a single AP. The distribution of heart potentials on the body surface depends not only on the sites of the excitation waves, but also on the geometry of the chest and the conductivity of body tissue. It is not surprising that overlap sites of potential minimum during the delta wave of the QRS complex were found in different locations of the AP. This means that the potential minimum alone cannot be a useful criterion for identifying the sites of the AP. If we take into consideration the direction of the excitation impulse as well as the location of the potential minimum, identification of AP sites will be improved. In our study, this combination of indices of BSPM clearly classified the 7 types of BSPM useful in identifying the sites of APs.

Epicardial RVBT is an indication of conduction through the AV node and His purkinje fiber. ${ }^{20)}$ Liebman et al. ${ }^{12)}$ did not find RVBT in their patients with $R V$ free wall or anteroseptal APs. In patients with the AP in the RV, the RV is not activated in the usual way by the specialized AV conduction system and the activation sequence of the RV is abnormal. Consequently, there is no RVBT. In patients with LV APs, however the activation of the RV develops via the specialized AV conduction system and results in a typical RVBT pattern. This was also true of the patients in the present study.

In eccentrically located dipole with radial direction, the surface potential maximum and potential minimum are located at the ends of a diameter with a higher absolute value at one extreme. ${ }^{6)}$ The greater strength of the anterior minimum as compared to the posterior maximum suggests that the pre-excitation wavefront was closer to the anterior chest surface than the posterior one. The location of pre-excitation could be either in the RV wall or in the right side of the interventricular septum. ${ }^{6}$ The abnormal early reversal pattern of ventricular depolarization was found in nearly all patients with RV APs in our study, demonstrating that this index is useful in differentiating between right-sided and leftsided APs.

Accordingly, the BSPM is a reliable, noninvasive procedure to determine 
the ventricular pre-excitation sites in patients with W-P-W syndrome. The following BSPM findings have been shown to be useful in identifying sites of APs: (1) the location of the potential minimum and direction of excitation wavefront during early ventricular depolarization; (2) epicardial RVBT; and (3) abnormal early reversal pattern of ventricular depolarization.

\section{REFERENCES}

1. Durrer D, Schuilenburg RM, Wellens HJJ. Pre-excitation revisited. Am J Cardiol 1970; 25: 690-7.

2. Sealy WC, Gallagher JJ, Pritchett EL. The surgical anatomy of Kent bundles based on electrophysiological mapping and surgical exploration. J Thorac Cardiovasc Surg 1978; 76: 804-15.

3. Taccardi B. Distribution of heart potentials on the thoracic surface of normal human subjects. Circ Res 1963; 12: 341-52

4. Tseng YZ, Tseng CD, Lo HM, Chiang FT, Hsu KL, Wu TL. Body surface potential maps of ventricular depolarization in normal adults. Jpn Heart J 1993; 34: 159-70.

5. Yamada K, Toyama J, Wada M, et al. Body surface isopotential mapping in Wolff-Parkinson-White syndrome. Am Heart J 1975; 90: 721-34.

6. De Ambroggi L, Taccardi B, Macchi E. Body surface maps of heart potentials: tentative localization of pre-excited areas in forty-two Wolff-Parkinson-White patients. Circulation 1976; 54: 251-63.

7. Oguri H, Wada $\mathrm{M}$, Sugenoya J, et al. Body surface potential distributions in posterior ventricular preexcitation. J Electrocardiol 1979; 12: 187-95.

8. Iwa T, Magara T. Correlation between localization of accessory conduction pathway and body surface maps in the Wolff-Parkinson-White syndrome. Jpn Circ J 1981; 45: 1192-8.

9. Benson W, Sterba R, Gallagher JJ, Walston II A, Spach MS. Localization of the site of ventricular pre-excitation with body surface maps in patients with Wolff-Parkinson-White syndrome. Circulation 1982; 65: 1259-68.

10. Groenewegen AS, Spekhorst HHM, Reek EJ. A quantitative method for the localization of the ventricular pre-excitation area in the Wolff-Parkinson-White syndrome using singular value decomposition of body surface potentials. J Electrocardiol 1985; 18: 157-68.

11. Kamakura S, Shimomura K, Ohe T, Matsuhisa M, Toyoshima H. The role of initial minimum potentials on body surface maps in predicting the site of accessory pathways in patients with WolffParkinson-White syndrome. Circulation 1986; 74: 89-96.

12. Liebman J, Zeno JA, Olshansky B, et al. Electrocardiographic body surface potential mapping in the Wolff-Parkinson-White syndrome: noninvasive determination of the ventricular insertion sites of accessory atrioventricular connections. Girculation 1991; 83: 886-901.

13. Dubuc M, Nadeau R, Tremblay G, Kus T, Molin F, Savard P. Pace mapping using body surface potential maps to guide catheter ablation of accessory pathways in patients with Wolff-Parkinson-White syndrome. Circulation 1993; 87: 135-43.

14. Giorgi C, Nadeau R, Savard P, Shenasa M, Page PL, Cardinal R. Body surface isopotential mapping of the entire QRST complex in the Wolff-Parkinson-White syndrome: correlation with the location of the accessory pathway. Am Heart J 1991; 121: 1445-53.

15. Hirai M, Tsuboi $N$, Hayashi $H$, et al. Body surface distribution of abnormally low $Q R S T$ areas in patients with Wolff-Parkinson-White syndrome: evidence for continuation of repolarization abnormalities before and after catheter ablation. Circulation 1993; 88: 2674-84.

16. Lamothe MJ, Stroin KG, Gardner MJ. Body surface potential mapping of a patient with WolffParkinson-White syndrome with two accessory pathways and two atrial pacemaker complexes. $J$ Electrocardiol 1996; 29: 139-47.

17. Tomita Y, Hirai M, Yanagawa $T$, et al. Body surface distribution of significant changes in QRST time-integral values after radiofrequency catheter ablation in patients with Wolff-Parkinson-White syndrome. Am J Cardiol 1996; 77: 59-63. 
18. Toyama J, Ohta T, Yamada K. Newly developed body surface mapping system for clinical use. In: Yamada K, Harumi K, Musha T, editors. Advances in Body Surface Potential Mapping. Nagoya: The University of Nagoya Press, 1983: 125-33.

19. Wilson FN, Johnston FD, Rosenbaum FF, Barker PS. On Eithoven's triangle, the theory of unipolar electrocardiographic leads, and the interpretation of the precordial electrocardiogram. Am Heart J 1946; 32: 277-310

20. Myerberg RJ, Nillson $\mathrm{K}$, Gelband $\mathrm{H}$. Physiology of the canine intraventricular conduction and endocardial excitation. Circ Res 1972; 30: 217-43. 